\title{
Protective effects of triptolide on retinal ganglion cells in a rat model of chronic glaucoma
}

This article was published in the following Dove Press journal:

Drug Design, Development and Therapy

16 November 2015

Number of times this article has been viewed

\section{Fan Yang \\ Dongmei Wang \\ Lingling Wu \\ Ying Li}

Ophthalmology Department, Peking University Third Hospital, Beijing,

People's Republic of China
Correspondence: Lingling Wu

Ophthalmology Department, Peking University Third Hospital, 49 Hua Yuan

Bei Lu, Hai Dian District, Beijing, People's Republic of China

$\mathrm{Tel}+8601082266562$

Fax +86 0108208 995I

Email wullc@hotmail.com
Purpose: To study the effects of triptolide, a Chinese herb extract, on retinal ganglion cells (RGCs) in a rat model of chronic glaucoma.

Methods: Eighty Wistar rats were randomly divided into triptolide group $(n=40)$ and normal saline (NS) group $(n=40)$. Angle photocoagulation was used to establish the model of glaucoma, with right eye as laser treated eye and left eye as control eye. Triptolide group received triptolide intraperitoneally daily, while NS group received NS. Intraocular pressure (IOP), anti-CD11b immunofluorescent stain in retina and optic nerve, RGCs count with Nissel stain and microglia count with anti-CD11b immunofluorescence stain in retina flat mounts, retinal tumor necrosis factor (TNF)- $\alpha$ mRNA detection by reverse transcription-polymerase chain reaction, and double immunofluorescent labeling with anti-TNF- $\alpha$ and anti-CD11b in retinal frozen section were performed.

Results: Mean IOP of the laser treated eyes significantly increased 3 weeks after photocoagulation $(P<0.05)$, with no statistical difference between the two groups $(P>0.05)$. RGCs survival in the laser treated eyes was significantly improved in the triptolide group than the NS group $(P<0.05)$. Microglia count in superficial retina of the laser treated eyes was significantly less in the triptolide group $(30.40 \pm 4.90)$ than the NS group $(35.06 \pm 7.59)(P<0.05)$. TNF- $\alpha$ mRNA expression in the retina of the laser treated eyes in the triptolide group decreased by $60 \%$ compared with that in the NS group $(P<0.01)$. The double immunofluorescent labeling showed that TNF- $\alpha$ was mainly distributed around the microglia.

Conclusion: Triptolide improved RGCs survival in this rat model of chronic glaucoma, which did not depend on IOP decrease but might be exerted by inhibiting microglia activities and reducing TNF- $\alpha$ secretion.

Keywords: glaucoma, triptolide, microglia, neuroprotection

\section{Background}

Glaucoma is the second most common cause of blindness in the world. ${ }^{1}$ It is a group of eye diseases with the common features of progressive apoptosis of retinal ganglion cells (RGCs), optic atrophy, and visual field defect. The development of glaucoma is closely related to the increased intraocular pressure (IOP); however, many other factors are involved in RGCs damage in glaucoma, and the pathogenic mechanism is not clear. Clinically, the IOP decrease therapy alone cannot achieve a satisfactory result; therefore, it is imperative to develop new therapies for inhibiting the apoptosis of RGCs and protecting the optic nerve.

Microglia is one of the family members of monocyte-macrophage cell system and widely distributed in the central nervous system $(\mathrm{CNS})$ and the retina. It plays important roles such as support, nutrition, immune response, repair, pro-regeneration, and others in the survival and the entire living activities of neurons. ${ }^{2}$ Microglia under homeostatic state has small size, with ramified branches and weak expression of cellular markers. Under pathological conditions, proliferation and morphological changes occur in 
microglia. It becomes an amoeba-like cell with enlarged cell body, fewer stubby branches, and strong expression of various cellular markers. ${ }^{3}$ The activated microglia may secrete inflammatory cytokines, proteolytic enzymes, and reactive oxygen species and induce the apoptosis of ganglion cells. ${ }^{4-7}$ Activated microglia has been found in the optic nerve in glaucoma patients and in the retina and optic nerve of animal models. ${ }^{8-11}$ Previous studies showed that the death of RGCs was associated with microglia and the inhibition of microglia activation could reduce the secretion of toxic cytokines and improve the survival of RGCs. ${ }^{10,12}$

Triptolide is one of the active ingredients extracted from the traditional Chinese medicine Celastraceae Tripterygium. It has the biological activities of anti-inflammation and immunosuppression, and it is mainly used for the treatment of rheumatoid arthritis, glomerulonephritis, and other autoimmune diseases. ${ }^{13,14}$ In vitro and in vivo trials have confirmed that triptolide can inhibit the activation of microglia, reduce the secretion of inflammatory cytokines, ${ }^{13-16}$ and improve neurons survival, ${ }^{17-19}$ suggesting its potential neuroprotective function.

The studies of using triptolide in treating glaucoma or the degenerative diseases of retina and optic nerve have been rarely reported. Our previous study is the only investigation that observed the protection effect of triptolide on RGCs by inhibiting the activation of microglia in a DBA/2J mouse model of glaucoma. ${ }^{20}$ However, DBA/2J mice are inherited pigmentary glaucoma model with high variability between animals. To confirm the effect of triptolide on RGCs and explore the mechanism, this study was performed on another glaucoma model. In this study, the method of aqueous release combined with angle photocoagulation was used to establish the rat model of chronic glaucoma, which could simulate the pathological process of chronic angle-closure glaucoma in a better way. ${ }^{21}$

\section{Materials and methods}

\section{Animals and groups}

Eighty Wistar rats of clean grade, 2 months old, weighing 200-250 g, were purchased from Vital River Laboratory Animal Technology Co Ltd (Beijing, People's Republic of China). The rats were housed in the Animal Department of Peking University Health Science Center and fed with coarse particle feed of clean grade and clean water in the conditions set at room temperature $\left(15^{\circ} \mathrm{C}-25^{\circ} \mathrm{C}\right)$ and 12 -hour light/12-hour dark schedule. Examinations prior to the trial showed clear cornea of both eyes and no lens opacities. The rats were randomly divided into the triptolide group $(n=40)$ and the normal saline (NS) group $(n=40)$ with a random digits table. The right eye of each rat was assigned as the "laser treated eye" and the left eye as the "control eye." The Association for Research in Vision and Ophthalmology statement was followed throughout the study. This study was approved by the ethical committee of Peking University Third Hospital. The experiments were performed in compliance with the National Institutes of Health Guide for Care and Use of Laboratory Animals.

\section{Dosing regimen}

Twenty milligrams of standard triptolide powder (purity $>99 \%$, purchased from National Institute for Food and Drug Control) was dissolved in $1 \mathrm{~mL}$ dimethyl sulfoxide solution and stored in $100 \mu \mathrm{L}$ aliquots at $-20^{\circ} \mathrm{C}$ for later use. Then, the working solution of $0.1 \mathrm{~g} / \mathrm{L}$ was prepared by dilution with NS. The rats in the triptolide group received triptolide $5 \mu \mathrm{g} / \mathrm{kg}$ intraperitoneally daily for 3 days before photocoagulation until euthanasia; the rats in the NS group received NS-diluted dimethyl sulfoxide solution with the same volume and concentration.

\section{Establishment of glaucoma animal model}

The method of aqueous release combined with angle photocoagulation was used to establish the rat model of chronic glaucoma. The right eyes served as the laser treated eyes, and the left eyes as the control eyes. Prior to the photocoagulation, tropicamide was used for mydriasis, and the mixture of ketamine $(50 \mathrm{mg} / \mathrm{kg})$ and xylazine $(5 \mathrm{mg} / \mathrm{kg})$ was given by intraperitoneal injection. After full anesthesia, the anterior chamber of the right eye was flattened by release of aqueous humor with a $26 \mathrm{G}$ microneedle. A multiwavelength laser (NOVUS OMNI, Lumenis Inc., Yokneam, Israel) was used to photocoagulate on corneoscleral limbus, with the laser range of $360^{\circ}$, the number of light spots of 41-85, and the following laser parameters: green, wavelength: $532 \mathrm{~nm}$, spot diameter: $200 \mu \mathrm{m}$, time: 0.5 seconds, and power: $0.38-0.50 \mathrm{~W}$. Then, photocoagulation was performed on three superficial veins at lateral temporal, superior temporal, and inferior temporal (3-6 spots for each blood vessel). The atropine ointment was applied once after photocoagulation to facilitate peripheral anterior synechiae.

\section{Anterior segment examination and IOP measurement}

After full anesthesia, the rats received anterior segments examination under a slit lamp. Then, the IOP was measured with the Tono-Pen XL tonometer. The average of four independent readings $(s<20)$ was recorded. All the measurements of IOP were performed between $9-10$ am by the same operator. 


\section{Nissel stain for retina flat mounts and RGC count}

The rats were sacrificed by anesthesia with an overdose of $30 \%$ urethane. After cardiac perfusion with $4 \%$ paraformaldehyde (PFA), the eyeballs were removed and fixed in $4 \%$ PFA solution for 1 hour at $4{ }^{\circ} \mathrm{C}$ and, after removing the anterior segment of the eye, it was fixed for another 7 hours. The retina was removed from the microscope and cut into four pieces radially. Then, the retina was tiled on a slide and coverslipped, and the slides were kept overnight under the pressure of a $10 \mathrm{~g}$ weight at room temperature. After removing the weight and the cover slip, $100 \mu \mathrm{L}$ of $1 \%$ cresyl violet solution was dropped onto the tissue on the slide. The slides were kept for 2 hours at $37^{\circ} \mathrm{C}$ and immersed in phosphate-buffered saline (PBS) for three times by 10 minutes per time. After dehydration with alcohol, transparency by xylene, and mounting with resin, the slides were observed under light microscope.

\section{RGCs count}

According to various cell morphological characteristics in RGC layer after the Nissel stain, the determination criterion of RGC was as follows: 1) round and large cell body; 2) clear nucleus and nucleolus; and 3) abundant cytoplasm with Nissel granule. The cells having the above features would be counted as RGC. The amacrine cells with small and round cell bodies and uniformly dark blue cytoplasm should not be counted; the cells with spindle-shaped cell bodies were commonly vascular endothelial cells and pericytes that should not be counted either. According to the distance from the disk, the retina is divided into three concentric regions, and each retina was divided radically into four sections. For each section of the retina, three visual fields were chosen, each in a different concentric region, thus, a total of 12 visual fields for each retina. An experienced lab assistant who was blinded to the grouping state counted the RGCs with Viewfinde Lite $(1.0 .134 \mathrm{c})$ program under optical microscope $(\times 1,000)$ (DP50; Olympus Corporation, Tokyo, Japan).

\section{Anti-CD I lb immunofluorescence staining for retina flat mounts and microglia count}

The eyeballs of the rats were removed, the retina was cut into four pieces for retina flat mounts, and then the specimens were fixed in ethanol for 10 minutes at $4^{\circ} \mathrm{C}$. After PBS immersion, the retina flat mounts were soaked in $0.1 \%$ Triton X-100 and $8 \%$ bovine serum albumin solution for 1 hour at room temperature. Then the mouse anti-rat $\mathrm{CD} 11 \mathrm{~b}$ antibodies were added (1:5), which will be kept overnight at $4^{\circ} \mathrm{C}$ and then for another 3 hours at $37^{\circ} \mathrm{C}$. After washing in PBS, the tetramethyl rhodamine isothiocyanate (TRITC)-labeled anti-mouse antibodies (1:200) were added and incubated overnight at $4^{\circ} \mathrm{C}$ and for another 3 hours at $37^{\circ} \mathrm{C}$; after PBS immersion, the retina flat mounts were mounted with antifluorescent quencher and observed under confocal microscope (Carl Zeiss LSM510; Carl Zeiss Meditec AG, Jena, Germany).

\section{Microglia count}

According to the distance from the disk, the retina is divided into three concentric regions, and each retina was divided radically into four sections. For each section of the retina, three visual fields were chosen, each in a different concentric region, thus, a total of 12 visual fields for each retina. A lab assistant who was blinded to the grouping state and the manifestation of the eyes took the pictures of the retina flat mounts and counted the microglia in the superficial layer of the retina.

\section{Preparation of frozen sections}

The rats were anesthetized with $30 \%$ urethane. After cardiac perfusion with $4 \%$ PFA, the eyeballs were removed and fixed in $4 \%$ PFA for 1 hour at $4^{\circ} \mathrm{C}$ and continued to fix overnight after the anterior segment was removed. After dehydration in $30 \%$ sucrose solution, the tissue blocks were embedded and stored at $-80^{\circ} \mathrm{C}$. Then the eyeball was cut into sections of $7 \mu \mathrm{m}$ along the sagittal axis with a freezing microtome. After fixed with acetone, the sections were stored at $-20^{\circ} \mathrm{C}$.

\section{H\&E stain for retinal frozen sections}

The frozen sections were stained in the hematoxylin solution for 5 minutes and rinsed with water. The color separation process was performed in the $\mathrm{HCl}$-alcoholic solution and terminated with water. The bluing process was performed in the ammonia solution and terminated with water. Then the sections were immersed in eosin solution for 5 minutes and rinsed with water. After dehydration with gradient alcohol, transparency by xylene, and mounting with resin, the slides were observed under light microscope (DP50).

\section{Double-staining immunofluorescence of CDIIb and tumor necrosis factor- $\alpha$ in retina frozen sections}

The frozen sections were blocked by goat serum for 30 minutes, then the mouse anti-rat $\mathrm{CD} 11 \mathrm{~b}$ antibodies (1:50) were added and incubated overnight at $4^{\circ} \mathrm{C}$. Then the TRITC-labeled anti-mouse immunoglobulin $\mathrm{G}(\operatorname{IgG})$ (1:200) was added and incubated for 1 hour at $37^{\circ} \mathrm{C}$ after PBS immersion. The rabbit anti-rat $\operatorname{IgG}$ (1:200) was added and incubated for 3 hours 
at room temperature after PBS immersion. The fluorescein isothiocyanate-labeled anti-rabbit $\operatorname{IgG}(1: 200)$ was added and incubated for 1 hour at $37^{\circ} \mathrm{C}$ after PBS immersion. Then the sections were mounted with antifluorescent quencher and observed under confocal microscope (Carl Zeiss LSM510).

\section{Total RNA extraction from retina}

A $500 \mu \mathrm{L}$ Trizol and a tissue block from the retina were added into a small amount of liquid nitrogen. After rapid grinding, the tissue block was transferred to $1.5 \mathrm{~mL}$ EP tube. Then, $100 \mu \mathrm{L}$ chloroform was added into the tube. After vigorous shaking, the tube was kept at room temperature. After centrifugation at $12,000 \mathrm{~g} / \mathrm{rpm}$ for 15 minutes at $4^{\circ} \mathrm{C}$, the upper aqueous phase was drawn and transferred to another centrifuge tube. Then, an equal volume of isopropanol was added and mixed thoroughly. After centrifugation at 12,000 g/rpm for 15 minutes at $4^{\circ} \mathrm{C}$, RNA pellet appeared. Then, the supernatant was discarded, and $1 \mathrm{~mL}$ of $75 \%$ alcohol prepared with RNase-free water was added to wash the RNA pellet. After centrifugation at $7,500 \mathrm{~g} / \mathrm{rpm}$ for 5 minutes at $4^{\circ} \mathrm{C}$, the supernatant was discarded. Once dried, $20 \mu \mathrm{L}$ of diethylpyrocarbonate water was added to dissolve the pellet and the product was temporarily stored at $-80^{\circ} \mathrm{C}$. After dilution of $3 \mu \mathrm{L}$ RNA by 1:40, the optical density (OD) value was measured with an UV spectrophotometer at $260 \mathrm{~nm}$ and $280 \mathrm{~nm}$, and the total concentration of RNA was calculated. Another $3 \mu \mathrm{L}$ RNA was used for $1 \%$ agarose gel electrophoresis.

\section{Reverse transcription-polymerase chain reaction}

1) M-MLV method was used to generate the first strand cDNA by reverse transcription (RT). Oligo (dT) $1 \mu \mathrm{L}$ was added, then diluted to $12 \mu \mathrm{L}$ with diethylpyrocarbonate water, mixing thoroughly; after simple centrifugation for 5 minutes at $65^{\circ} \mathrm{C}$, placed on ice for 2 minutes and added $5 \times$ reaction buffer $4 \mu \mathrm{L}$, RNA inhibitor $(20 \mu / \mu \mathrm{L}) 1 \mu \mathrm{L}, 10 \mathrm{mM}$ deoxy-ribonucleoside triphosphate mix $2 \mu \mathrm{L}$ and M-MLV reverse transcriptase $(200 \mu / \mu \mathrm{L}) 1 \mu \mathrm{L}$, with the total volume of $20 \mathrm{~L}$, mixing thoroughly; after simple centrifugation for 60 minutes at $42^{\circ} \mathrm{C}$, incubated for 5 minutes at $70^{\circ} \mathrm{C}$ to terminate the reaction. 2) Polymerase chain reaction (PCR): tumor necrosis factor (TNF)- $\alpha$ primer: upstream primer 5-CGTCGTAGCAAACCACCAAG-3, downstream primer 5-CACAGAGCAATGACTCCAAAG-3, $\beta$-actin primers: upstream primer 5-AGGCCAACCGTGAAAAGATG-3, downstream primer 5-ACCAGAGGCATACAGGGACAA-3. Sequentially added: $10 \mu \mathrm{L}$ PCR mixture (Tiangen), $7 \mu \mathrm{L}$ RNase-free water, water, $2 \mu \mathrm{L}$ cDNA, $1 \mu \mathrm{L}$ working solution of primer; $94^{\circ} \mathrm{C}$ ( 5 minutes $), 94^{\circ} \mathrm{C}$ (30 seconds), annealing temperature ( 30 seconds), $72^{\circ} \mathrm{C}$ ( 30 seconds), $72^{\circ} \mathrm{C}$ (10 minutes), a total of 30 cycles. 3 ) PCR products underwent agarose gel electrophoresis at $120 \mathrm{~V}$ for 40 minutes and then the OD of each electrophoresis band was scanned. The relative expression level of TNF- $\alpha$ mRNA was calculated as OD of the electrophoresed band of the TNF- $\alpha$ RT-PCR product/ internal reference OD of the RT-PCR products of $\beta$-actin.

\section{Statistical methods}

Statistical analysis was performed using SPSS software (version 13.0). The data of the laboratory tests were presented by $\bar{x} \pm s$, and the data in each group showed homogeneity of variance with Levene test. The paired $t$-test was used to compare the values of the IOP, RGCs, microglia, and TNF- $\alpha$ mRNA between the laser treated eyes and the control eyes at the same point in the same group; independent-samples $t$-test was used to compare the values of IOP, RGCs, microglia, and TNF- $\alpha$ mRNA of the laser treated eyes at the same point between the groups. $P<0.05$ was considered to be statistically significant.

\section{Results}

The rat model of chronic glaucoma was established with the method of aqueous release combined with angle photocoagulation in a total of 80 rats of the triptolide group $(n=40)$ and the NS group $(n=40)$. Two rats that died unexpectedly in anesthesia before laser treatment, two rats that had their lens damaged during puncture of anterior chamber, and one rat that had endophthalmitis were all excluded from the study. A total of 75 rats completed the study, 38 rats in the triptolide group and 37 rats in the NS group. Photographs of anterior segment after photocoagulation are shown in Figure 1.

\section{IOP}

A total of 16 rats in both groups (eight rats for each group) received IOP measurements of both eyes with a Tonopen XL tonometry on Day 1 before laser photocoagulation and on Days 1, 3, 5, and 7 after laser photocoagulation and then once weekly until euthanasia.

There was no significant difference between the mean pre-photocoagulation IOPs of right eye and left eye in both the groups $(P>0.05)$. Since Day 1 after photocoagulation, the IOP of the laser treated eyes in the NS group was significantly increased compared with that before photocoagulation and reached the peak value on Week 1 . Then, the hypertension continuously lasted for $\sim 3$ weeks and recovered on Week 4. For the control eyes, the IOP at each point after photocoagulation did not differ from that before photocoagulation (Table 1). There was no statistical significant difference in the IOP between the laser treated eyes in the NS group and 

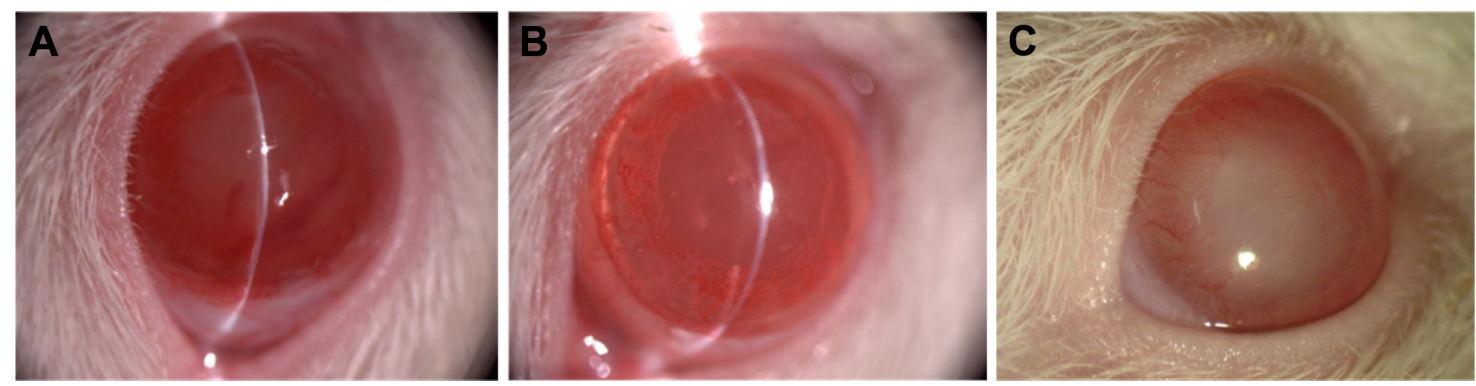

Figure I Photographs of anterior segment after photocoagulation.

Notes: (A) One day after photocoagulation, conjunctiva hyperemia, corneal edema, and anterior chamber disappeared. (B) Three days after photocoagulation, cornea recovered and transparent, iris vessels, and anterior chamber recovered. (C) Five days after laser surgery, cataract developed.

that in the triptolide group at each point after photocoagulation $(P>0.05)$ (Table 1). The IOP change of the laser treated eyes in the triptolide group was basically consistent with that in the NS group (Figure 2).

\section{Change of RGCs}

\section{H\&E staining of retinal frozen sections}

$H \& E$ staining was performed on the retinal frozen sections of the laser treated eyes in both the groups before photocoagulation and on Weeks 1, 2, 4, and 8 after photocoagulation (Figure 3). The number of blue-stained nuclei in the RGC layer of the laser treated eyes in the NS group was gradually decreased over time (Figure 3B-E). However, little change was observed over time in the triptolide group (Figure 3F-I).

\section{Nissel staining of retina flat mounts}

Nissel staining of retina flat mounts was performed in both eyes of the rats in the two groups (24 rats each group) at the time point of Weeks 1, 2, 4, and 8 after photocoagulation, and a statistical comparison was done for the numbers of RGCs. In the NS group, the Nissel bodies in RGCs in the laser treated eyes decreased or disappeared after photocoagulation and the light blue-stained RGCs gradually decreased over time (Figure 4). The loss of RGCs began from Week 1 and reached a peak within Weeks 4-8 after photocoagulation (Table 2). Whereas the RGC count in the laser treated eyes of the triptolide group did not show significant decrease compared with that in the control eyes ( $P>0.5$; Table 2$)$. At each time point after photocoagulation, the RGCs count in the laser treated eyes in the triptolide group was significantly more than that in the NS group $(P<0.05$; Table 2$)$.

\section{Changes of retina and optic nerve microglia}

Immunofluorescence for microglia in frozen sections of retina

Eight weeks after photocoagulation, the anti-CD11b staining was performed in the retinal frozen sections of both eyes in the two groups (Figure 5A-D). The red stained cells were the microglia, and it was found that microglia in the control eyes of both groups were in homeostatic state: small cell bodies, lots of ramified branches, stained lightly and uniformly (Figure 5A and $\mathrm{C}$ ). The microglia in the laser treated eyes of the NS group showed activated state: the cell bodies were larger, with less and stubby branches, dark staining (Figure 5B), and the number of microglia increased compared with that in control eyes, mainly distributed in the superficial layer of retina: nerve fiber layer, ganglion cell layer, and inner plexiform layer. In the triptolide group, some microglia in the laser treated eyes showed activated state, while others showed homeostatic state, suggesting a slight activation (Figure 5D).

\section{Immunofluorescence for microglia in frozen sections of optic nerve}

Eight weeks after photocoagulation, the anti-CD11b staining in the frozen sections of the optic nerve was performed in the two groups (Figure 5E-H). The optic nerve microglia in the control eyes were lightly stained, with small cell bodies and ramified branches (Figure 5E and G). The optic nerve microglia showed darkly stained cytosome, fewer and thicker branches, some even showed granular shapes in the laser treated eyes of the NS group, and the number of microglia increased compared with that in the control eyes (Figure 5F). However, the optic nerve microglia in the laser treated eyes of the triptolide group still showed small and lightly stained cell bodies with ramified branches, presenting homeostatic states (Figure $5 \mathrm{H}$ ).

\section{Immunofluorescence for microglia in retina flat mounts}

Eight weeks after photocoagulation, anti-CD11b immunofluorescence staining was performed in the retina flat mounts of both eyes in the two groups (six rats each group) (Figure 5I-L). Microglia in the control eyes showed homeostatic states (Figure 5I and K). Their hollow cell bodies with dendritic 


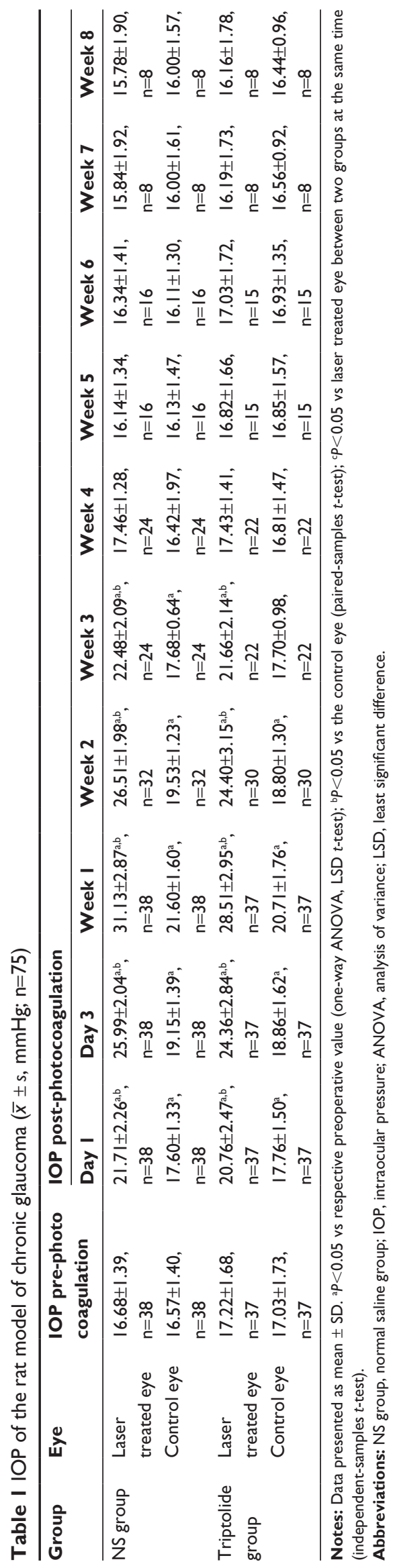

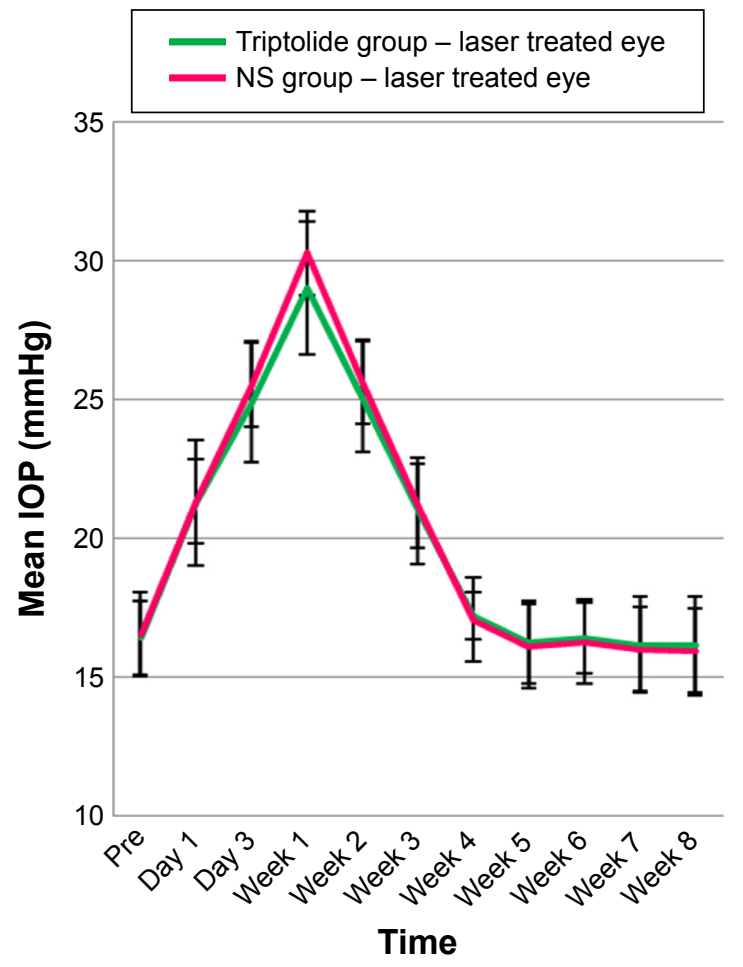

Figure 2 Mean IOP of the rats in the NS group and triptolide group. Abbreviations: IOP, intraocular pressure; NS, normal saline.

prominence presented stretching forms and uniformly distributed in the superficial layer of the retina. Many homeostatic state microglia tangled on the large vessels in the superficial layer of the retina. Microglia in the laser treated eyes of the NS group showed activated amoeboid profile (Figure 5J). Their cell bodies became larger, showing solid appearance, darkly stained, less, and shorter prominences. The number of microglia also increased significantly compared with the control eyes $(P>0.05)$ (Table 3). Whereas the microglia of the laser treated eyes in the triptolide group were partially activated (Figure $5 \mathrm{~L}$ ). The microglia number $(30.40 \pm 4.90)$ in the superficial layer of the retina for the laser treated eyes in the triptolide group significantly decreased compared with (35.06 \pm 7.59$)$ that in the laser treated eyes in the NS group $(P<0.05)$ (Table 3$)$.

\section{Change of TNF- $\alpha$ expression}

\section{Anti-TNF- $\alpha$ immunofluorescence staining in retinal} frozen sections

Anti-TNF- $\alpha$ immunofluorescence staining was performed in the retinal frozen sections of both groups 8 weeks after photocoagulation (Figure 6). The immunofluorescence intensity and area of the TNF- $\alpha$ in the laser treated eyes of the NS group (Figure 6B) increased obviously compared with the control eyes (Figure 6A) and TNF- $\alpha$ was mainly distributed in the inner retinal layers: including nerve fiber 

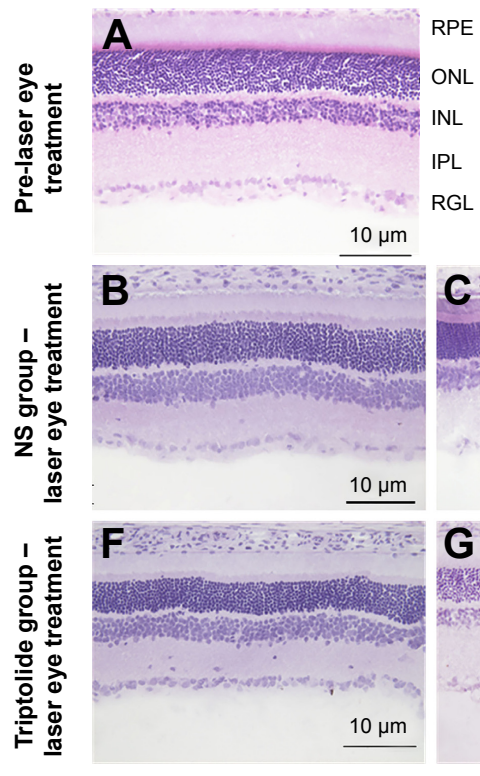

Week 1
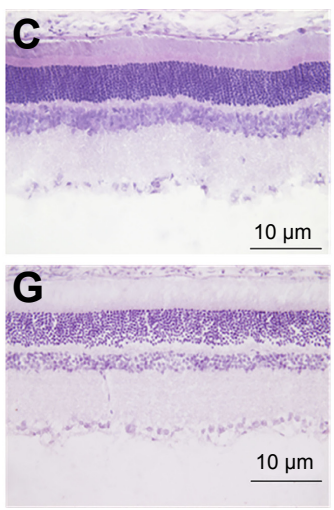

Week 2
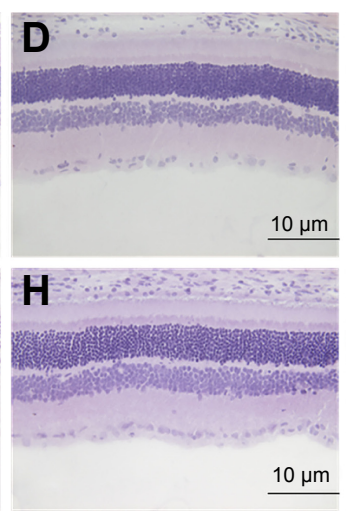

Week 4
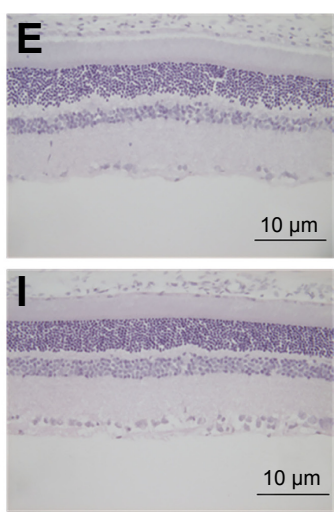

Week 8

Figure $3 \mathrm{H} \& \mathrm{E}$ staining of the retinal frozen sections of the laser treated eyes in the two groups.

Notes: (A) The normal retina before photocoagulation, with lots of nuclei in retina ganglion cell layer (RGL). After photocoagulation, the number of nuclei in RGL in the NS group gradually decreased over time (B-E), while little change was observed over time in the triptolide group (F-I).

Abbreviations: H\&E, hematoxylin and eosin; NS group, normal saline group; RPE, retinal pigment epithelium; ONL, outer nuclear layer; INL, inner nuclear layer; IPL, inner plexiform layer; RGL, retinal ganglion cell layer.

layer, ganglion cell layer, and inner plexiform layer. Little difference was observed for the positive expression of TNF- $\alpha$ in the laser treated eyes of the triptolide group (Figure 6D) compared with that in the control eyes (Figure 6C). However, there was an obvious reduction of immunofluorescence intensity and area of the TNF- $\alpha$ in the laser treated eyes of the triptolide group (Figure 6D) compared with the laser treated eyes of the NS group (Figure 6B).
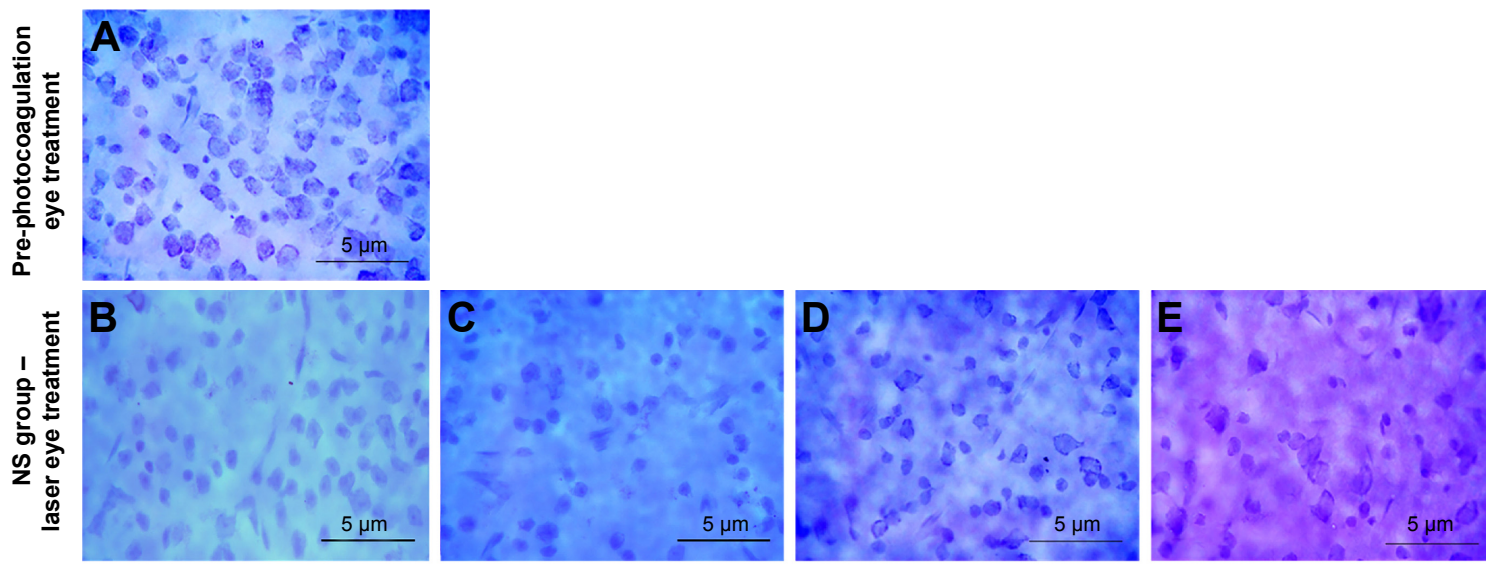

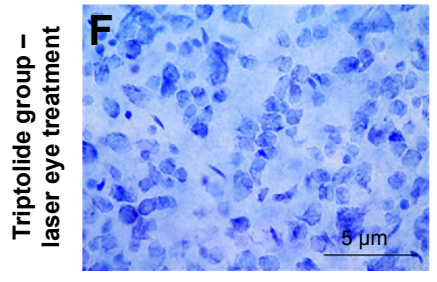

Week 1

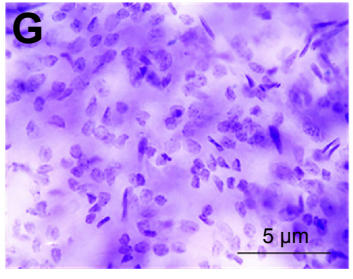

Week 2

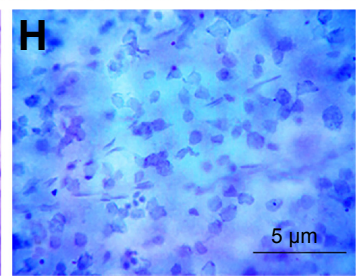

Week 4

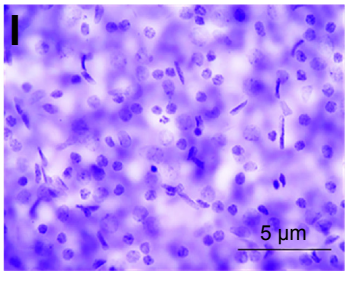

Week 8

Figure 4 Nissel staining of the retina flat mount of the laser treated eyes in the two groups.

Notes: A shows the RGCs in the retina of pre-photocoagulation eye. The number of RGCs in laser treated eyes in the NS group (B-E) decreased gradually over time, and only a few of them were visible at Week 8; whereas, at the same time point, the RGCs count in the laser treated eyes in the triptolide group was more than that in the NS group (F-I) RGCs count did not reduce significantly.

Abbreviations: RGCs, retinal ganglion cells; NS group, normal saline group. 
Table 2 RGC count of the rat model of chronic glaucoma $\left(\bar{x} \pm s\right.$, cells $\left./ 0.024 \mathrm{~mm}^{2} ; \mathrm{n}=48\right)$

\begin{tabular}{llllll}
\hline Group & Eye & \multicolumn{3}{l}{ RGC count post-photocoagulation } & \\
\cline { 2 - 6 } & & Week I & Week 2 & Week 4 & Week 8 \\
\hline NS group & Laser treated eye & $59.67 \pm 5.68^{\mathrm{a}, \mathrm{b}}$ & $58.83 \pm 7.67^{\mathrm{a}, \mathrm{b}}$ & $56.89 \pm 6.90^{\mathrm{a}, \mathrm{b}}$ & $46.06 \pm 12.34^{\mathrm{a}-\mathrm{c}}$ \\
& Control eye & $67.33 \pm 4.76$ & $67.25 \pm 6.30$ & $67.22 \pm 13.32$ & $68.17 \pm 10.93$ \\
Triptolide group & Laser treated eye & $68.17 \pm 9.28$ & $66.67 \pm I 1.79$ & $67.17 \pm 6.24$ & $66.22 \pm 7.72$ \\
& Control eye & $67.00 \pm 6.42$ & $68.33 \pm 12.25$ & $68.58 \pm 7.96$ & $67.28 \pm 13.29$ \\
\hline
\end{tabular}

Notes: Data presented as mean \pm SD. a $P<0.05$ vs the laser treated eyes in the triptolide group (independent-samples $t$-test); ${ }^{b}<0.05$ vs the control eye in the same group (paired-samples $t$-test); ${ }^{c}>0.01$ vs the laser treated eyes at different weeks in the NS group (one-way ANOVA, LSD $t$-test).

Abbreviations: NS group, normal saline group; RGC, retinal ganglion cell; ANOVA, analysis of variance; LSD, least significant difference.

\section{RT-PCR for TNF- $\alpha$ mRNA in retina}

Total mRNA was extracted from the retina of four rats in each group 8 weeks after photocoagulation, and a semiquantitative detection performed after RT-PCR amplification revealed that the expression of TNF- $\alpha$ in the retina of the laser treated eyes in the NS group was 2.4-fold more than that in the control group $(P<0.01)$ (Figures 7 and 8 ). For the laser treated eyes in the triptolide group, no marked change was seen for the expression of TNF- $\alpha$ mRNA compared with the control eyes, and a reduction of $60 \%$ was observed compared with the laser treated eyes in the NS group $(P<0.01)$ (Figures 7 and 8).

\section{Double immunofluorescent labeling for retinal microglia and TNF- $\alpha$}

Double immunofluorescent labeling with anti-TNF- $\alpha$ and anti-CD11b in the retinal frozen section was performed
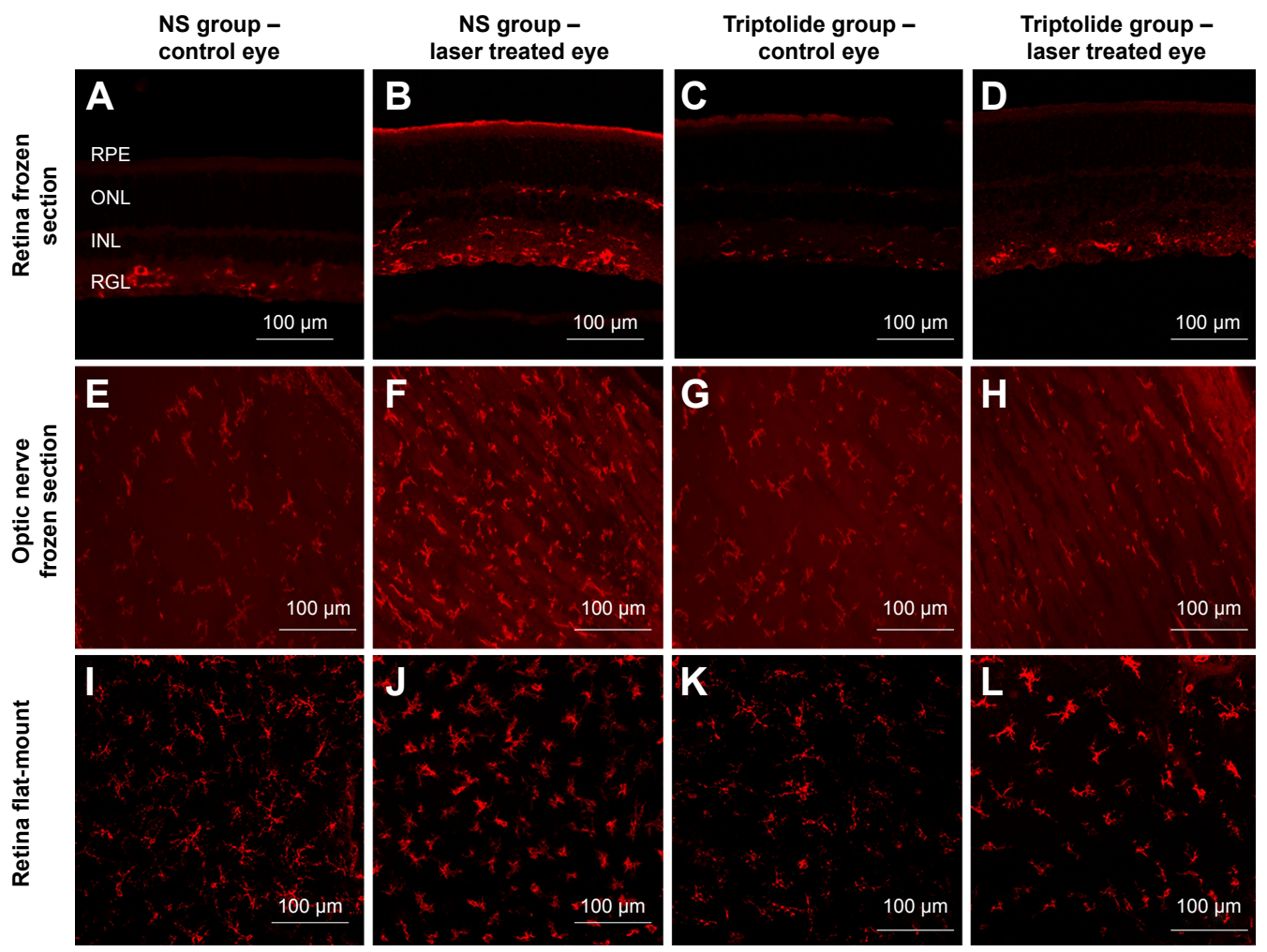

Figure 5 Anti-CDI lb staining 8 weeks after photocoagulation.

Notes: 8 weeks after photocoagulation, the microglia in control eyes of the two groups presented homeostatic state: small cell bodies, multigrade ramified branches, and similar immunoreactive intensity $(\mathbf{A}, \mathbf{C}, \mathbf{E}, \mathbf{G}, \mathbf{I}, \mathbf{K})$. Most of the microglia in the laser treated eyes in the NS groups showed activated state: larger cell bodies, fewer and thicker branches, and darker staining (B, F, J). A few of the microglia in the laser treated eyes in the triptolide groups showed activated state, while the others showed homeostatic state ( $\mathbf{D}, \mathbf{H}, \mathbf{L})$. Retinal microglia of the laser treated eyes in both groups was mainly distributed in the superficial layer of the retina: nerve fiber layer, ganglion cell layer, and inner plexiform layer (B, D), and the microglia density in the laser treated eyes in the triptolide group (D) was less prominent than that in the NS group (B). A-D: anti-CDI lb staining on retinal frozen sections 8 weeks after photocoagulation. $\mathbf{E}-\mathbf{H}$ : anti-CDIIb staining on frozen sections of optic nerve 8 weeks after photocoagulation. I-L: anti-CDI Ib staining on frozen sections of retina flat mounts 8 weeks after photocoagulation. A, E, I: NS group, control eyes; B, F, J: NS group, laser treated eyes; C, G, K: triptolide group, control eyes; D, H, L: triptolide group, laser treated eyes.

Abbreviations: NS group, normal saline group; RPE, retinal pigment epithelium; ONL, outer nuclear layer; INL, inner nuclear layer; IPL, inner plexiform layer; RGL, retinal ganglion cell layer. 
Table 3 Retina microglia count 8 weeks after photocoagulation $(\bar{x} \pm s$, cells/I2 field; $\mathrm{n}=\mid 2)$

\begin{tabular}{lll}
\hline Group & Control eye & Laser treated eye \\
\hline NS group & $27.89 \pm 5.14$ & $35.06 \pm 7.59^{\mathrm{a}, \mathrm{b}}$ \\
Triptolide group & $23.00 \pm 7.76$ & $30.40 \pm 4.90^{\mathrm{b}}$ \\
\hline
\end{tabular}

Notes: Data presented as mean \pm SD. ${ }^{a} P<0.05$ vs the laser-treated eyes in the triptolide group (independent-samples $t$-test); ${ }^{\mathrm{b}} \mathrm{P}<0.05$ vs the control eye (pairedsamples $t$-test)

Abbreviation: NS group, normal saline group.

8 weeks after photocoagulation in the laser treated eyes of the NS group, and it was revealed that TNF- $\alpha$ in the retina (TRITC-labeled anti-TNF- $\alpha$ ) was mainly distributed around the microglia (fluorescein isothiocyanate-labeled anti-CD11b) (Figure 9).

\section{Discussion}

Triptolide, a diterpenoid epoxide extracted from the traditional Chinese medicine Tripterygium wilfordii Hook F (molecular structure shown in Figure 10), has been used for a long time with the immunosuppressive, anti-inflammatory, and anti-proliferative effects. ${ }^{13,14}$ Due to the pharmacological properties of small molecular weight and lipophilicity, triptolide can penetrate the blood-brain barrier. ${ }^{13,14}$ Accordingly, triptolide has been widely used in the studies of the neurodegenerative diseases in CNS and has proven its neuroprotective function. ${ }^{18,19,22}$ Glaucoma is an ocular neurodegenerative disease similar to the neurodegenerative diseases in CNS. The retina also has a blood-retina barrier similar to that in the CNS, so conventional medication therapies have difficulty achieving desired concentration in the retina. Therefore, this study transferred the methods of treating the CNS disorders to the study of glaucoma.

The rat model of chronic glaucoma established by laser photocoagulation in this study simulated glaucomatous pathological properties of chronic intraocular hypertension and progressive loss of RGC. The results of IOP and loss of RGCs in the NS group were consistent with the findings of Levkovitch-Verbin et al. ${ }^{21}$ The RGC count in the laser treated eyes in the triptolide group did not show significant difference compared with that in the control eyes, whereas the RGC count was significantly more than that in the laser treated eyes of the NS group (Table 2), indicating that triptolide could improve the survival of RGCs in this chronic glaucoma model. In the study of inherited pigmentary glaucoma in DBA/2J mouse model,
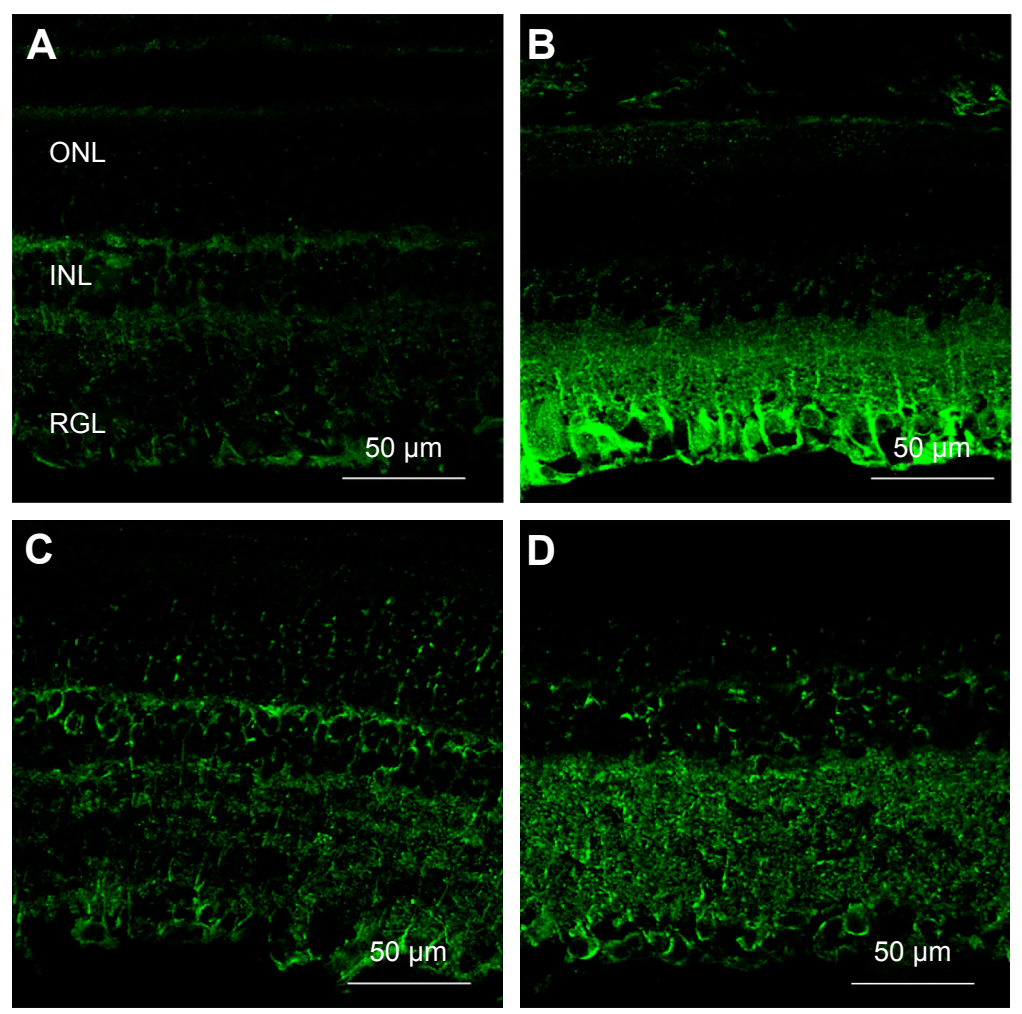

Figure 6 Anti-TNF- $\alpha$ staining on frozen retinal sections 8 weeks after photocoagulation.

Notes: The FITC-labeled sections were anti-TNF- $\alpha$ staining. Strong positive expression can be observed in the inner layer of retina in the laser treated eye in the NS group (B) and weak expression in the control eye (A). The expression of TNF- $\alpha$ in the laser treated eye in the triptolide group (D) was weaker than that in the NS group (B). A: NS group, control eyes; B: NS group, laser treated eyes; C: triptolide group, control eyes; $\mathbf{D}$ : triptolide group, laser treated eyes.

Abbreviations: TNF, tumor necrosis factor; NS group, normal saline group; ONL, outer nuclear layer; INL, inner nuclear layer; RGL, retinal ganglion cell layer. 


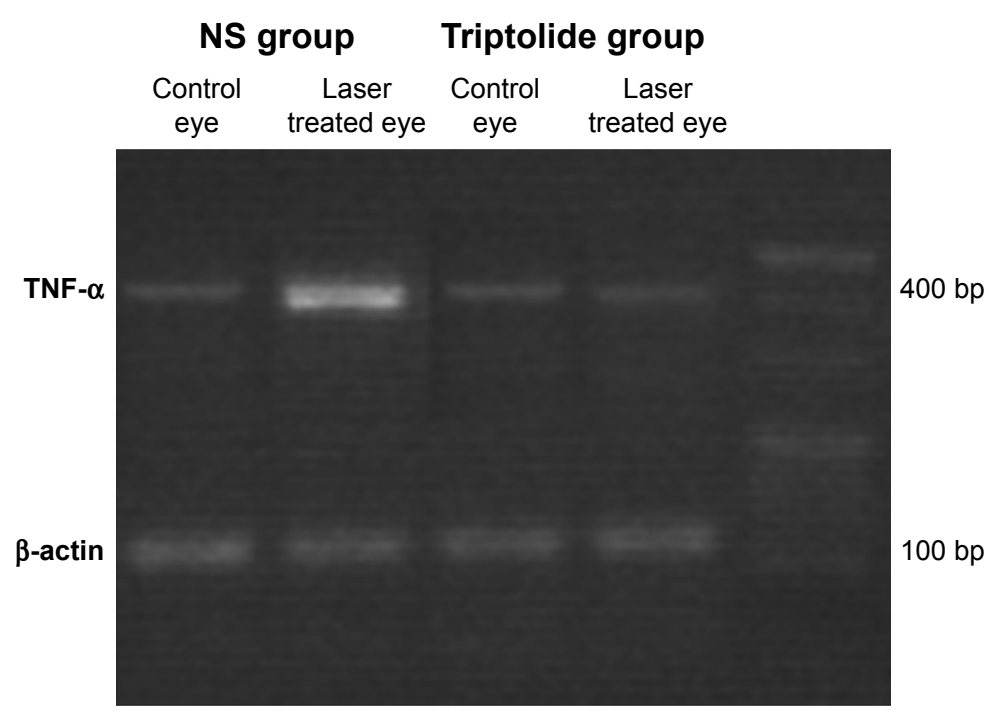

Figure 7 Expression of TNF- $\alpha$ mRNA 8 weeks after photocoagulation.

Notes: The expression of TNF- $\alpha$ mRNA in the laser treated eyes in the NS group increased significantly compared with the control eyes. The expression of TNF- $\alpha$ mRNA in the laser treated eyes in the triptolide group was not significantly changed compared with the control eyes.

Abbreviations: NS group, normal saline group; TNF, tumor necrosis factor.

the protective effect of triptolide on the RGCs had also been manifested. ${ }^{20}$ Although there was no report about the effect of triptolide on the production and outflow of aqueous humor as far as we know, the previous study on DBA/2J mice found that IOP in the triptolide group did not differ significantly compared with the control group. ${ }^{20}$ Also in this study, no statistical significant difference was detected in the IOP of laser treated eyes between the two groups at any point after photocoagulation (Figure 2), indicating that the protective function of triptolide on RGCs was not achieved by the reduction of IOP.

Microglia of the control eyes in the NS group showed homeostatic state, simultaneously, prominent proliferation

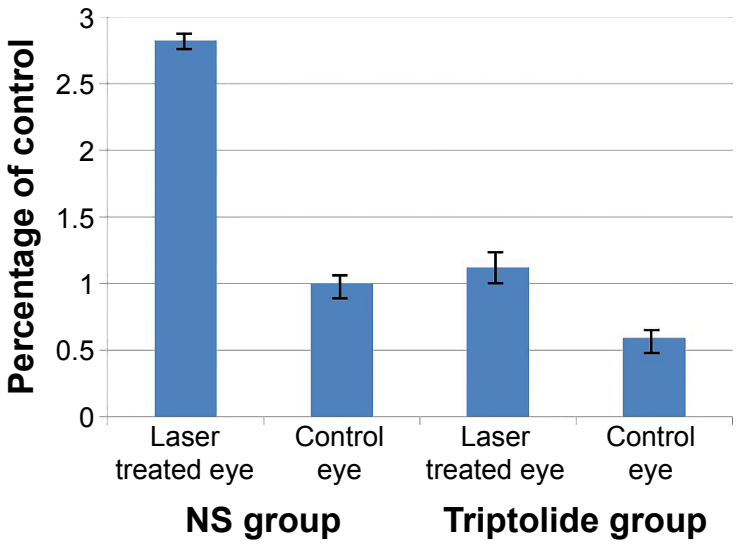

Figure 8 Relative gray value of TNF- $\alpha$ mRNA 8 weeks after photocoagulation. Notes: The expression of TNF- $\alpha$ mRNA in the laser treated eyes of the retina in the NS group increased significantly compared with that in the control eyes $(P<0.0 \mathrm{I})$. Significant reduction in the expression of TNF- $\alpha$ mRNA was observed in the retina of the laser treated eyes in the triptolide group compared to the NS group $(P<0.01)$. Abbreviations: NS, normal saline; TNF, tumor necrosis factor. and activation of the microglia occurred in the retina and optic nerve of the laser treated eyes in the NS group induced by the high IOP after photocoagulation (Figure 5). The activated retinal microglia mainly distributed in the superficial layer of the retina which was closely related with RGCs. However, due to triptolide administration, retinal microglia count significantly decreased and activation of microglia was depressed in the laser treated eyes of the triptolide group (Figure 5; Table 3). This indicated that triptolide inhibited the activation of microglia: reducing proliferation, inhibiting morphological changes, and downregulating the expression of cell markers. Many previous studies in vitro and in vivo also showed that triptolide could inhibit the activation of microglia in CNS and played a role in protecting neurons. ${ }^{16-19,22}$

The immunofluorescence intensity and area of the TNF- $\alpha$ in the laser treated eyes of the NS group increased obviously compared with control, indicating increased production of TNF- $\alpha$ in the retina of this glaucoma model (Figure 6). The intensive TNF- $\alpha$, as an inflammatory cytokine, mainly distributed in the inner layers of the retina near the activated retinal microglia and RGCs. So we supposed that TNF- $\alpha$ might originate from activated microglia and threaten RGCs survival. Another important evidence to support this hypothesis was the colocalization of retinal microglia and TNF- $\alpha$ in double immunofluorescent staining, which indicated that retinal microglia might synthesize and secrete TNF- $\alpha$ in the situation of high IOP (Figure 9). It has been demonstrated that RGCs express the TNF- $\alpha$ receptor 1 that can promote the apoptosis of these cells, so it is reasonable to believe that 

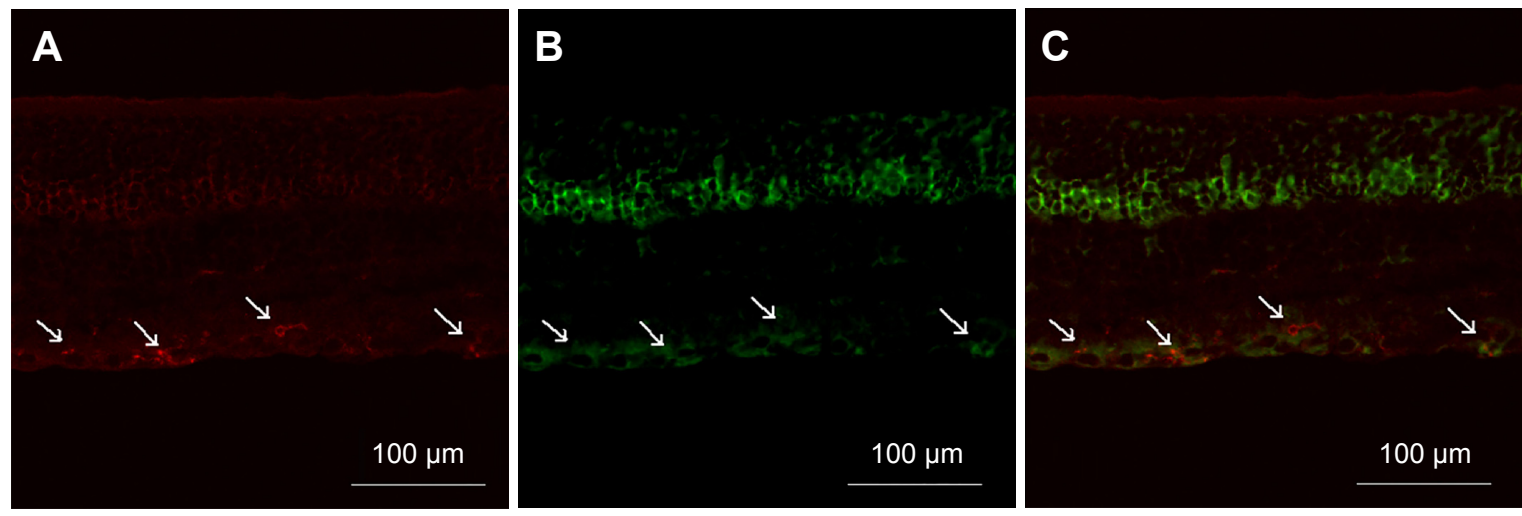

Figure 9 Double immunofluorescent staining of anti-TNF- $\alpha$ and anti-CDIlb in retinal frozen sections of the laser treated eyes in the normal saline group 8 weeks after photocoagulation.

Notes: TRITC-labeled anti-CDI Ib staining (A), FITC-labeled anti-TNF- $\alpha$ staining (B), and double staining labeled colocalization site (C). CDII Ib and TNF- $\alpha$ were mainly distributed in the superficial layers of the retina: nerve fiber layer, ganglion cell layer, and inner plexiform layer. TNF- $\alpha$ mainly distributed around the microglia $(C D I I b[+])$ and the colocalization of them was observed. The arrows in A show positive staining of CDIIb. The arrows in B show positive staining of TNF- $\alpha$. The arrows in C show positive double staining of CDI Ib and TNF- $\alpha$.

Abbreviations: TNF, tumor necrosis factor; FITC, fluorescein isothiocyanate.

RGCs are very sensitive to TNF- $\alpha .{ }^{23}$ Many previous studies also inferred that TNF- $\alpha$ might play a role in promoting the death of RGCs in glaucoma and found increased TNF- $\alpha$ expression in the glaucoma patients. ${ }^{24-29}$ Furthermore, Bosco et $\mathrm{al}^{10}$ pointed out that microglia began to become activated earlier than the apoptosis of RGCs at the early period of optic neuropathy of glaucoma. Nakazawa et $\mathrm{al}^{30}$ found that TNF- $\alpha$ could induce the activation of microglia. So we speculated that in this rat model of chronic glaucoma, the increased IOP induced by photocoagulation might induce microglia to become activated, and TNF- $\alpha$ secreted by these activated microglia not only caused RGCs death and subsequent optic nerve degeneration but also promoted the activation of the surrounding microglia, which was a positive feedback process. This process would further promote continuous activation of microglia even after discontinuation of the primary stimulating factors and partly explained the continuous activation of microglia and the progressive apoptosis of RGCs after IOP decrease.

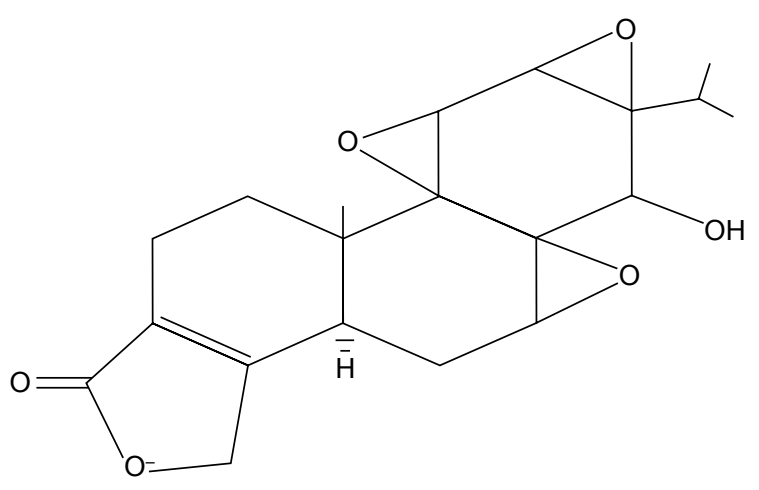

Figure 10 Molecular structure of triptolide.
In this study, there was an obvious reduction of TNF- $\alpha$ immunofluorescence intensity and area in the laser treated eyes of the triptolide group compared with that in the NS group (Figure 6), suggesting that TNF- $\alpha$ expression was restrained by triptolide. Further evidence was that RT-PCR amplification revealed a $60 \%$ reduction of TNF- $\alpha$ mRNA in the laser treated eyes in the triptolide group compared with that in the NS group (Figures 7 and 8). This result was consistent with previous studies that triptolide could reduce the secretion of inflammatory cytokines such as TNF- $\alpha$ from CNS microglia in vitro and in vivo. ${ }^{15,17,19}$ So, triptolide was suspected to protect RGCs in this glaucoma model by inhibiting retinal microglia from producing inflammatory cytokines, such as TNF- $\alpha$.

The immunosuppressive and anti-inflammatory mechanisms of triptolide are mainly due to its inhibition effect on the inflammatory cytokines, such as the synthesis and secretion of TNF- $\alpha$, interleukin- $1 \beta, \mathrm{COX}-2$, and so on. ${ }^{15-17}$ Gong et $\mathrm{a}^{16}$ reported that the anti-inflammatory mechanism of triptolide was probably mediated by inhibiting the transcriptional activity of microglia nuclear factor kappa B (NFkappaB), and NF-kappaB was also an important factor in the binding of TNF- $\alpha$ to its receptor and its downstream signals. Therefore, it was supposed that microglia might be the target cells of triptolide for its activities. Although the immunosuppressive and anti-inflammatory effects of triptolide may also inhibit other immune cells, such as lymphocytes, dendritic cells, and macrophages, the blood-retinal barrier can impede above mentioned immune cells from the circulation entering the retina and the subsequent immune response. ${ }^{31}$ However, microglia, as innate immune cells of CNS, may 
be the important target cells of triptolide in the retina of this rat model of glaucoma. Therefore, we hypothesized that the protective effect of triptolide on RGCs might be achieved through activating microglia and reducing the secretion of inflammatory factors.

It is an important strategy to treat neurodegenerative diseases by regulating immune response of microglia and inhabitating its toxicity. ${ }^{32}$ Bosco et $\mathrm{al}^{12}$ used minocycline, an inhibitor of microglia, in DBA/2J mouse; it was manifested that RGC survival and RGC axonal transport and integrity were improved. In this study, the use of triptolide also follows the strategy of microglia inhabitation. Of course, in addition to effective pharmacological effects, there are also many reports of side effects and adverse reactions for triptolide, including liver and kidney toxicity and reproductive toxicity. ${ }^{33,34}$ Although TNF- $\alpha$ level of control eyes in the NS group was higher than that of the triptolide group, which raised the concern of the toxicity effect of triptolide on microglia, it could not come to a conclusion that triptolide had toxicity effect to microglia, because retinal microglia in the control eyes of the triptolide group showed normal appearance in homeostatic state and their number did not decrease significantly compared with that of the NS group. Due to the limited conditions in this study, dose response and toxicity effect experiments of triptolide were not conducted in this study; however, we chose the treatment concentration and dose according to Zhou et al's report, ${ }^{19}$ as the concentration and dose were relatively low in all the other reports about triptolide. The pharmacodynamic study was also not conducted in this study. Although we have not found any report involving the distribution and concentration of triptolide in the retina after systemic administration, many studies have shown that triptolide can pass through the blood-brain barrier and can be used in the treatment of CNS diseases. ${ }^{18,19,22}$ The results of this study have proved that triptolide has protective effect on RGCs that leads us to believe that triptolide can achieve effective concentration in retina; however, it requires further studies to prove.

\section{Conclusion}

Triptolide can improve the survival of RGCs in the rat model of chronic glaucoma. This protective effect on RGCs does not depend on the decrease of IOP but may be exerted by inhibiting the activities of microglia and the reduction in the secretion of TNF- $\alpha$.

\section{Disclosure}

The authors report no conflicts of interest in this work.

\section{References}

1. Quigley HA, Broman AT. The number of people with glaucoma worldwide in 2010 and 2020. Br J Ophthalmol. 2006;90(3):262-267.

2. Streit WJ. Microglia as neuroprotective, immunocompetent cells of the CNS. Glia. 2002;40(2):133-139.

3. Streit WJ, Walter SA, Pennell NA. Reactive microgliosis. Prog Neurobiol. 1999;57(6):563-581.

4. Wang JW, Chen SD, Zhang XL, Jonas JB. Retinal microglia in glaucoma. J Glaucoma. Epub 2015 Feb 23.

5. Mcguire SO, Ling ZD, Lipton JW, Sortwell CE, Collier TJ, Carvey PM. Tumor necrosis factor alpha is toxic to embryonic mesencephalic dopamine neurons. Exp Neurol. 2001;169(2):219-230.

6. Glass CK, Saijo K, Winner B, Marchetto MC, Gage FH. Mechanisms underlying inflammation in neurodegeneration. Cell. 2010;140(6): 918-934.

7. Ransohoff RM, Perry VH. Microglial physiology: unique stimuli, specialized responses. Annu Rev Immunol. 2009;27:119-145.

8. Yuan L, Neufeld AH. Activated microglia in the human glaucomatous optic nerve head. J Neurosci Res. 2001;64(5):523-532.

9. Madeira MH, Boia R, Santos PF, Ambrósio AF, Santiago AR. Contribution of microglia-mediated neuroinflammation to retinal degenerative diseases. Mediators Inflamm. 2015;2015:673090.

10. Bosco A, Steele MR, Vetter ML. Early microglia activation in a mouse model of chronic glaucoma. J Comp Neurol. 2011;519(4):599-620.

11. Naskar R, Wissing M, Thanos S. Detection of early neuron degeneration and accompanying microglial responses in the retina of a rat model of glaucoma. Invest Ophthalmol Vis Sci. 2002;43(9):2962-2968.

12. Bosco A, Inman DM, Steele MR, et al. Reduced retina microglial activation and improved optic nerve integrity with minocycline treatment in the DBA/2J mouse model of glaucoma. Invest Ophthalmol Vis Sci. 2008;49(4):1437-1446.

13. Shui G, Wan Y, Jiang C, et al. Progress in Tripterygium wilfordiiand its bioactive components in the field of pharmacodynamics and pharmacology. Zhong Guo Zhong Yao Za Zhi. 2010;35(4):515-520.

14. Brinker AM, Ma J, Lipsky PE, Raskin I. Medicinal chemistry and pharmacology of genus triptergium (celastraceae). Phytochemistry. 2007; 68(6):732-766.

15. Jiao J, Xue B, Zhang L, et al. Triptolide inhibits amyloid-beta1-42induced TNF-alpha and IL-1beta production in cultured rat microglia. J Neuroimmunol. 2008;205(1-2):32-36.

16. Gong Y, Xue B, Jiao J, Jing L, Wang X. Triptolide inhibits COX-2 expression and PGE2 release by suppressing the activity of NF-kappaB and JNK in LPS-treated microglia. J Neurochem. 2008;107(3): 779-788.

17. Zhou HF, Niu DB, Xue B, et al. Triptolide inhibits TNF-alpha, IL-1 beta and NO production in primary microglial cultures. Neuroreport. 2003;14(7):1091-1095.

18. Lu L, Li F, Wang X. Novel anti-inflammatory and neuroprotective agents for Parkinson's disease. CNS Neurol Disord Drug Targets. 2010; 9(2):232-240.

19. Zhou HF, Liu XY, Niu DB, Li FQ, He QH, Wang XM. Triptolide protects dopaminergic neurons from inflammation-mediated damage induced by lipopolysaccharide intranigral injection. Neurobiol Dis. 2005;18(3):441-449.

20. Yang F, Wu LL, Guo XJ, Wang D, Li Y. Improved retinal ganglion cell survival through retinal microglia suppression by a Chinese herb extract, triptolide, in the DBA/2J mouse model of glaucoma. Ocul Immunol Inflamm. 2013;21(5):378-389.

21. Levkovitch-Verbin H, Quigley HA, Martin KR, Valenta D, Baumrind LA, Pease ME. Translimbal laser photocoagulation to the trabecular meshwork as a model of glaucoma in rats. Invest Ophthalmol Vis Sci. 2002;43(2):402-410.

22. Li FQ, Lu XZ, Liang XB, et al. Triptolide, a Chinese herbal extract, protects dopaminergic neurons from inflammation-mediated damage through inhibition of microglial activation. J Neuroimmunol. 2004; 148(1-2):24-31. 
23. Fontaine V, Mohand-Said S, Hanoteau N, Fuchs C, Pfizenmaier K, Eisel U. Neurodegenerative and neuroprotective effects of tumor necrosis factor (TNF) in retinal ischemia: opposite roles of TNF receptor 1 and TNF receptor 2. J Neurosci. 2002;22:RC216.

24. Križaj D, Ryskamp DA, Tian N, et al. From mechanosensitivity to inflammatory responses: new players in the pathology of glaucoma. Curr Eye Res. 2014;39(2):105-119.

25. Yuan L, Neufeld AH. Tumor necrosis factor-alpha: a potentially neurodestructive cytokine produced by glia in the human glaucomatous optic nerve head. Glia. 2000;32(1):42-50.

26. Tezel G, Li LY, Patil RV, Wax MB. TNF-alpha and TNF-alpha receptor-1 in the retina of normal and glaucomatous eyes. Invest Ophthalmol Vis Sci. 2001;42(8):1787-1794.

27. Al-Gayyar MM, Elsherbiny NM. Contribution of TNF- $\alpha$ to the development of retinal neurodegenerative disorders. Eur Cytokine Netw. 2013; 24(1):27-36.

28. Tezel G. TNF-alpha signaling in glaucomatous neurodegeneration. Prog Brain Res. 2008;173:409-421.
29. Tezel G, Wax MB. Increased production of tumor necrosis factor-alpha by glial cells exposed to simulated ischemia or elevated hydrostatic pressure induces apoptosis in cocultured retinal ganglion cells. J Neurosci. 2000;20(23):8693-8700.

30. Nakazawa T, Nakazawa C, Matsubara A, et al. Tumor necrosis factoralpha mediates oligodendrocyte death and delayed retinal ganglion cell loss in a mouse model of glaucoma. J Neurosci. 2006;26(49): 12633-12641.

31. Qiu D, Kao PN. Immunosuppressive and anti-inflammatory mechanisms of triptolide, the principal active diterpenoid from the Chinese medicinal herb Tripterygium wilfordii Hook. f. Drugs R D. 2003;4(1):1-18.

32. Choi DK, Koppula S, Suk K. Inhibitors of microglial neurotoxicity: focus on natural products. Molecules. 2011;16(2):1021-1043.

33. Ni B, Jiang Z, Huang X, et al. Male reproductive toxicity and toxicokinetics of triptolide in rats. Arzneimittelforschung. 2008;58(12):673-680.

34. Liu L, Jiang Z, Liu J, et al. Sex differences in subacute toxicity and hepatic microsomal metabolism of triptolide in rats. Toxicology. 2010;271(1-2):57-63

\section{Publish your work in this journal}

Drug Design, Development and Therapy is an international, peerreviewed open-access journal that spans the spectrum of drug design and development through to clinical applications. Clinical outcomes, patient safety, and programs for the development and effective, safe, and sustained use of medicines are a feature of the journal, which has also been accepted for indexing on PubMed Central. The manuscript management system is completely online and includes a very quick and fair peer-review system, which is all easy to use. Visit http://www.dovepress.com/testimonials.php to read real quotes from published authors.

Submit your manuscript here: http://www.dovepress.com/drug-design-development-and-therapy-journal 